\title{
Risk Management of Risk Under the Basel Accord: A Bayesian Approach to Forecasting Value-at-Risk of VIX Futures*
}

\author{
Roberto Casarin \\ Department of Economics \\ Ca’ Foscari University of Venice \\ Chia-Lin Chang \\ Department of Applied Economics \\ Department of Finance \\ National Chung Hsing University \\ Taichung, Taiwan \\ Juan-Ángel Jiménez-Martín \\ Department of Quantitative Economics \\ Complutense University of Madrid \\ Michael McAleer \\ Econometric Institute \\ Erasmus School of Economics \\ Erasmus University Rotterdam \\ and \\ Tinbergen Institute \\ The Netherlands \\ and \\ Institute of Economic Research \\ Kyoto University, Japan \\ Teodosio Pérez-Amaral \\ Department of Quantitative Economics \\ Complutense University of Madrid
}

EI 2011-29

Revised: July 2011

\footnotetext{
* The authors are most grateful for the helpful comments and suggestions of participants at the Kansai Econometrics Conference, Osaka, Japan, January 2011, and the International Conference on Risk Modelling and Management, Madrid, Spain, June 2011. For financial support, the second author acknowledges the National Science Council, Taiwan, the third and fifth authors acknowledge the Ministerio de Ciencia y Tecnología and Comunidad de Madrid, Spain, and the fourth author wishes to thank the Australian Research Council, National Science Council, Taiwan, and the Japan Society for the Promotion of Science.
} 


\begin{abstract}
It is well known that the Basel II Accord requires banks and other Authorized Deposit-taking Institutions (ADIs) to communicate their daily risk forecasts to the appropriate monetary authorities at the beginning of each trading day, using one or more risk models, whether individually or as combinations, to measure Value-at-Risk (VaR). The risk estimates of these models are used to determine capital requirements and associated capital costs of ADIs, depending in part on the number of previous violations, whereby realised losses exceed the estimated VaR. McAleer et al. (2009) proposed a new approach to model selection for predicting $\mathrm{VaR}$, consisting of combining alternative risk models, and comparing conservative and aggressive strategies for choosing between $\mathrm{VaR}$ models. This paper addresses the question of risk management of risk, namely VaR of VIX futures prices, and extends the approaches given in McAleer et al. (2009) and Chang et al. (2011) to examine how different risk management strategies performed during the 2008-09 global financial crisis (GFC). The empirical results suggest that an aggressive strategy of choosing the Supremum of single model forecasts, as compared with Bayesian and non-Bayesian combinations of models, is preferred to other alternatives, and is robust during the GFC. However, this strategy implies relatively high numbers of violations and accumulated losses, which are admissible under the Basel II Accord.
\end{abstract}

Keywords: Median strategy, Value-at-Risk, daily capital charges, violation penalties, aggressive risk management, conservative risk management, Basel Accord, VIX futures, Bayesian strategy, quantiles, forecast densities.

JEL Classifications: G32, G17, C53, C22, C11. 


\section{Introduction}

As has been discussed in many outlets (see, for example, Chang et al. (2011)), volatility derivatives have attracted a great deal of attention over the past years since they enable trading and hedging against changes in volatility. In 1993 the Chicago Board Options Exchange (CBOE) introduced a volatility index, VIX (see Whaley (1993)), which was originally designed to measure the market expectation of 30-day volatility implied by at-themoney S\&P100 option prices. The original volatility index is now known as VXO. In 2003, together with Goldman Sachs, CBOE updated and reformulated VIX to reflect a model-free method of measuring expected volatility, one that continues to be widely used by financial theorists.

Unlike the original VIX, the updated VIX is based on the S\&P500 index, rather than S\&P100 option prices, and estimates expected volatility by averaging the weighted prices of S\&P500 puts and calls over a wide range of strike prices. Although many market participants have considered the index to be a good predictor of short term volatility, daily or even intraday, it took several years for the market to introduce volatility products, starting with over-thecounter products, such as variance swaps. The first exchange-traded product, VIX futures, was introduced in March 2004, and was followed by VIX options in February 2006. Both of these volatility derivatives are based on the VIX index as the underlying asset.

Of the two most popular volatility derivatives, this paper focuses on VIX futures to address risk management of risk. McAleer, et al. (2009, 2010, 2011) analyse how the new market risk management strategies performed during the 2008-09 global financial crisis (GFC), and evaluate how the GFC affected the best risk management practices. Huskaj (2009) analyzes VIX futures from a different perspective, centered on the statistical properties of a different set of candidate forecasting models, and without focusing on the Basel II regulations, as is considered in this paper. McAleer and Wiphatthanananthakul (2010) examine the empirical behaviour of alternative simple expected volatility indexes, and compare them with VIX. Chang et al. (2011) analyse the VaR of VIX futures under the Basel Accord before, during and after the GFC.

The GFC has greatly affected economic and financial structures worldwide, and has led to numerous attempts to analyse the underlying causes, as well as to improve regulations 
governing financial markets and the associated risks. There have been many questions asked about whether appropriate regulations were in place, especially in the USA, which is not a signatory to the Basel Accords, to permit the appropriate monitoring and encouragement of (possibly excessive) risk taking.

As is well known, the Basel II Accord was designed to monitor and encourage sensible risk taking using appropriate models of risk to calculate Value-at-Risk (VaR) and the corresponding daily capital charges. When the Basel I Accord was concluded in 1988, no capital requirements were defined for market risk. However, regulators soon recognized the risks to a banking system if insufficient capital were held to absorb the large sudden losses from huge exposures in capital markets. During the mid-90's, proposals were tabled for an amendment to the 1988 Accord, requiring additional capital over and above the minimum required for credit risk. Finally, a market risk capital adequacy framework was adopted in 1995 for implementation in 1998.

As discussed in Chang et al. (2011), for example, the 1995 Basel I Accord amendment provides a menu of approaches for determining market risk capital requirements, ranging from simple to intermediate and advanced approaches. Under the advanced approach (that is, the internal model approach), banks are allowed to calculate the capital requirement for market risk using their internal models. The use of internal models was introduced in 1998 in the European Union. The 26 June 2004 Basel II framework, implemented in many countries in 2008 (though not yet in the USA), enhanced the requirements for market risk management by including, for example, oversight rules, disclosure, management of counterparty risk in trading portfolios.

VaR is now a standard tool in risk management, and is defined as an estimate of the probability and size of the potential loss to be expected over a given period. It has become especially important following the 1995 amendment to the Basel Accord, whereby banks and other Authorized Deposit-taking Institutions (ADIs) were permitted (and encouraged) to use internal models to forecast daily VaR (see Jorion (2000) for a detailed discussion). The last decade has witnessed a growing academic and professional literature comparing alternative modelling approaches to determine how to measure VaR, especially for large portfolios of financial assets. 
In order to encourage and reward institutions with superior risk management systems, amendments were made to modify the initial Basel Accord. A back-testing procedure, whereby actual returns are compared with the corresponding VaR forecasts, was introduced to assess the quality of the internal models used by ADIs. In cases where internal models lead to a greater number of violations than could reasonably be expected, given the confidence level, the ADI is required to hold a higher level of capital (see Table 1 for the penalties imposed under the Basel II Accord). Penalties imposed on ADIs affect profitability directly through higher capital charges, and indirectly through the imposition of a more stringent external model to forecast VaR. This is one reason why financial managers typically prefer risk management strategies that are passive and conservative rather than active and aggressive.

Excessive conservatism can have a negative impact on the profitability of ADIs as higher capital charges are subsequently required. Therefore, ADIs have been encouraged to consider a strategy that allows an endogenous decision as to how many times ADIs should violate in any financial year (for further details, see McAleer and da Veiga (2008a, 2008b), McAleer (2009), Caporin and McAleer (2009b), McAleer et al. (2009), and Chang et al. (2011)). This paper suggests alternative aggressive and conservative risk management strategies, including strategies based on Bayesian methodology, that can be compared with the use of one or more models of risk, whether individually or in combination, in the estimation and forecasting periods.

As an extension of Chang et al. (2011), this paper defines risk management in terms of choosing sensibly from a variety of risk models, discusses the selection of optimal risk models, considers combining alternative risk models, discusses the choice between conservative and aggressive (Bayesian and non-Bayesian) risk management strategies, evaluates the effects of the Basel II Accord on risk management of risk, examines how some risk management strategies performed during the 2008-09 GFC, and evaluates how the GFC affected risk management practices and daily capital charges.

The empirical results indicate that, when risk management is considered for VIX futures, the optimal strategy based on comparing individual and combined models of risk, is to be aggressive rather than conservative. Specifically, this would involve a strategy of communicating to the national regulatory authority the Supremum of the point forecasts of the VaR models considered. This strategy tends to minimize the average daily capital charges, 
subject to staying within the limits of the number of violations that are permitted under the Basel II Accord.

The remainder of the paper is organized as follows. In Section 2 we present the main ideas of the Basel II Accord Amendment as it relates to forecasting VaR and daily capital charges. Section 3 reviews some of the most well-known univariate models of conditional volatility that are used to forecast $\mathrm{VaR}$, including optimal Bayesian strategies based on combined models of risk. In Section 4 the data used for estimation and forecasting are presented. Section 5 analyses the VaR forecasts before, during and after the 2008-09 GFC. Section 6 presents some concluding remarks.

\section{Forecasting Value-at-Risk and Daily Capital Charges}

In this section, which closely follows Chang et al. (2011), we evaluate risk management of risk by applying the Basel II formulae to a period that includes the 2008-09 GFC. The Basel II Accord stipulates that daily capital charges (DCC) must be set at the higher of the previous day's VaR or the average VaR over the last 60 business days, multiplied by a factor $(3+k)$ for a violation penalty, wherein a violation involves the actual negative returns exceeding the VaR forecast negative returns for a given day:

$$
\mathrm{DCC}_{\mathrm{t}}=\sup \left\{-(3+\mathrm{k}) \overline{\mathrm{VaR}}_{60},-\mathrm{VaR}_{\mathrm{t}-1}\right\}
$$

where

$D C C_{t}=$ daily capital charges, which is the higher of $-(3+\mathrm{k}) \overline{\mathrm{VaR}}_{60}$ and $-\mathrm{VaR}_{\mathrm{t}-1}$,

$V a R_{t}=$ Value-at-Risk for day $t$,

$\operatorname{VaR}_{t}=\hat{Y}_{t}-z_{t} \cdot \hat{\sigma}_{t}$,

$\overline{\mathrm{VaR}}_{60}=$ mean $\mathrm{VaR}$ over the previous 60 working days,

$\hat{Y}_{t}=$ estimated return at time $\mathrm{t}$, 
$z_{t}=1 \%$ critical value of the distribution of returns at time $\mathrm{t}$,

$\hat{\sigma}_{t}=$ estimated risk (or square root of volatility) at time $\mathrm{t}$,

$0 \leq \mathrm{k} \leq 1$ is the Basel II violation penalty (see Table 1 ).

\section{[Insert Table 1 here]}

The formula given in equation (1) is contained in the 1995 amendment to Basel I, while Table 1 appears for the first time in the Basel II Accord in 2004. The multiplication factor (or penalty), $k$, depends on the central authority's assessment of the ADI's risk management practices and the results of a simple backtest. It is determined by the number of times actual losses exceed a particular day's VaR forecast (Basel Committee on Banking Supervision (1996, 2006)).

As discussed in Stahl (1997), the minimum multiplication factor of 3 is intended to compensate for various errors that can arise in model implementation, such as simplifying assumptions, analytical approximations, small sample biases and numerical errors that tend to reduce the true risk coverage of the model. Increases in the multiplication factor are designed to increase the confidence level that is implied by the observed number of violations at the 99\% confidence level, as required by regulators (for a detailed discussion of $\mathrm{VaR}$, as well as exogenous and endogenous violations, see McAleer (2009), Jiménez-Martin et al. (2009), and McAleer et al. (2010a)).

In calculating the number of violations, ADIs are required to compare the forecasts of VaR with realised profit and loss figures for the previous 250 trading days. In 1995, the 1988 Basel Accord (Basel Committee on Banking Supervision (1988)) was amended to allow ADIs to use internal models to determine their VaR thresholds (Basel Committee on Banking Supervision (1995)). However, ADIs that propose using internal models are required to demonstrate that their models are sound. Movement from the green zone to the red zone arises through an excessive number of violations. Although this will lead to a higher value of $k$, and hence a higher penalty, violations will also tend to be associated with lower daily capital 
charges. It should be noted that the number of violations in a given period is an important, though not the only, guide for regulators to approve a given VaR model.

VaR refers to the lower bound of a confidence interval for a (conditional) mean, that is, a "worst case scenario on a typical day". If interest lies in modelling the random variable, $Y_{t}$, it could be decomposed as follows:

$$
Y_{t}=E\left(Y_{t} \mid F_{t-1}\right)+\varepsilon_{t}
$$

This decomposition states that $Y_{t}$ comprises a predictable component, $E\left(Y_{t} \mid F_{t-1}\right)$, which is the conditional mean, and a random component, $\varepsilon_{t}$. The variability of $Y_{t}$, and hence its distribution, is determined by the variability of $\varepsilon_{t}$. If it is assumed that $\varepsilon_{t}$ follows a conditional distribution, such that:

$$
\varepsilon_{t} \sim D\left(\mu_{t}, \sigma_{t}^{2}\right)
$$

where $\mu_{t}$ and $\sigma_{t}$ are the conditional mean and standard deviation of $\varepsilon_{t}$, respectively, these can be estimated using a variety of parametric, semi-parametric or non-parametric methods.

The VaR threshold for $Y_{t}$ can be calculated as:

$$
\operatorname{VaR}_{t}=E\left(Y_{t} \mid F_{t-1}\right)-\alpha \sigma_{t}
$$

where $\alpha$ is the critical value from the distribution of $\varepsilon_{t}$ to obtain the appropriate confidence level. It is possible for $\sigma_{t}$ to be replaced by alternative estimates of the conditional standard deviation in order to obtain an appropriate $\mathrm{VaR}$ (for useful reviews of theoretical results for conditional volatility models, see Li et al. (2002) and McAleer (2005), where several univariate and multivariate, conditional, stochastic and realized volatility models are discussed). 
Some recent empirical studies (see, for example, Berkowitz and O’Brien (2001), Gizycki and Hereford (1998), and Pérignon et al. (2008)) have indicated that some financial institutions overestimate their market risks in disclosures to the appropriate regulatory authorities, which can imply a costly restriction to the banks trading activity. ADIs may prefer to report high VaR numbers to avoid the possibility of regulatory intrusion. This conservative risk reporting suggests that efficiency gains may be feasible. In particular, as ADIs have effective tools for the measurement of market risk, while satisfying the qualitative requirements, ADIs could conceivably reduce daily capital charges by implementing a context-dependent market risk disclosure policy. McAleer (2009), McAleer et al. (2010a) and Chang et al. (2011) discuss alternative approaches to optimize VaR and daily capital charges.

\section{Models for Forecasting VaR}

The following section extends the analysis in McAleer et al. (2010b) and Chang et al. (2011) to include optimal Bayesian strategies based on combined models of risk. It is well known that ADIs can use internal models to determine their VaR thresholds. There are alternative time series models for estimating conditional volatility. In what follows, we present several well-known conditional volatility models that can be used to evaluate strategic market risk disclosure, namely GARCH, GJR and EGARCH, with Gaussian, Student- $t$ and Generalized Normal distribution errors, where the parameters are estimated.

These models are chosen as they are widely used in the literature. For an extensive discussion of the theoretical properties of several of these models (see Ling and McAleer (2002a, 2002b, 2003a) and Caporin and McAleer (2010b)). As an alternative to estimating the parameters, we also consider the exponential weighted moving average (EWMA) method by Riskmetrics (1996) and Zumbauch, (2007) that calibrates the unknown parameters. We include a section on these models to present them in a unified framework and notation, and to make explicit the specific versions we are using. Apart from EWMA, the models are presented in increasing order of complexity. Optimal Bayesian strategies based on combined models of risk are also discussed.

\subsection{GARCH}


For a wide range of financial data series, time-varying conditional variances can be explained empirically through the autoregressive conditional heteroskedasticity (ARCH) model, which was proposed by Engle (1982). When the time-varying conditional variance has both autoregressive and moving average components, this leads to the generalized $\operatorname{ARCH}(p, q)$, or $\operatorname{GARCH}(p, q)$, model of Bollerslev (1986). It is very common in practice to impose the widely estimated GARCH(1,1) specification in advance.

Consider the stationary AR(1)-GARCH(1,1) model for daily returns, $y_{t}$ :

$$
y_{t}=\varphi_{1}+\varphi_{2} y_{t-1}+\varepsilon_{t}, \quad\left|\varphi_{2}\right|<1
$$

for $t=1, \ldots, n$, where the shocks to returns are given by:

$$
\begin{aligned}
\varepsilon_{t} & =\eta_{t} \sqrt{h_{t}}, \quad \eta_{t} \sim \operatorname{iid}(0,1) \\
h_{t} & =\omega+\alpha \varepsilon_{t-1}^{2}+\beta h_{t-1}
\end{aligned}
$$

and $\omega>0, \alpha \geq 0, \beta \geq 0$ are sufficient conditions to ensure that the conditional variance $h_{t}>0$. The stationary AR(1)-GARCH(1,1) model can be modified to incorporate a nonstationary $\operatorname{ARMA}(p, q)$ conditional mean and a stationary $\operatorname{GARCH}(r, s)$ conditional variance, as in Ling and McAleer (2003b).

\subsection{GJR}

In the symmetric GARCH model, the effects of positive shocks (or upward movements in daily returns) on the conditional variance, $h_{t}$, are assumed to be the same as the effect of negative shocks (or downward movements in daily returns) of equal magnitude. In order to accommodate asymmetric behaviour, Glosten, Jagannathan and Runkle (1992) proposed a model (hereafter GJR), for which GJR(1,1) is defined as follows:

$$
h_{t}=\omega+\left(\alpha+\gamma I\left(\eta_{t-1}\right)\right) \varepsilon_{t-1}^{2}+\beta h_{t-1}
$$

where $\omega>0, \alpha \geq 0, \alpha+\gamma \geq 0, \beta \geq 0$ are sufficient conditions for $h_{t}>0$, and $I\left(\eta_{t}\right)$ is an indicator variable defined by: 


$$
I\left(\eta_{t}\right)= \begin{cases}1, & \varepsilon_{t}<0 \\ 0, & \varepsilon_{t} \geq 0\end{cases}
$$

as $\eta_{t}$ has the same sign as $\varepsilon_{t}$. The indicator variable differentiates between positive and negative shocks, so that asymmetric effects in the data are captured by the coefficient $\gamma$. For financial data, it is expected that $\gamma \geq 0$ because negative shocks have a greater impact on risk than do positive shocks of similar magnitude. The asymmetric effect, $\gamma$, measures the contribution of shocks to both short run persistence, $\alpha+\gamma / 2$, and to long run persistence, $\alpha+\beta+\gamma / 2$.

Although GJR permits asymmetric effects of positive and negative shocks of equal magnitude on conditional volatility, the special case of leverage, whereby negative shocks increase volatility while positive shocks decrease volatility (see Black (1976) for an argument using the debt/equity ratio), cannot be accommodated, in practice (for further details on asymmetry versus leverage in the GJR model, see Caporin and McAleer (2010b)).

\subsection{EGARCH}

An alternative model to capture asymmetric behaviour in the conditional variance is the Exponential GARCH, or EGARCH(1,1), model of Nelson (1991), namely:

$$
\log h_{t}=\omega+\alpha\left|\frac{\varepsilon_{t-1}}{h_{t-1}}\right|+\gamma \frac{\varepsilon_{t-1}}{h_{t-1}}+\beta \log h_{t-1}, \quad|\beta|<1
$$

where the parameters $\alpha, \beta$ and $\gamma$ have different interpretations from those in the GARCH(1,1) and GJR(1,1) models.

EGARCH captures asymmetries differently from GJR. The parameters $\alpha$ and $\gamma$ in EGARCH(1,1) represent the magnitude (or size) and sign effects of the standardized residuals, respectively, on the conditional variance, whereas $\alpha$ and $\alpha+\gamma$ represent the effects of positive and negative shocks, respectively, on the conditional variance in GJR(1,1). 
Unlike GJR, EGARCH can accommodate leverage, depending on the restrictions imposed on the size and sign parameters, though leverage is not guaranteed (for further details, see Caporin and McAleer (2010b)).

As noted in McAleer et al. (2007), there are some important differences between EGARCH and the previous two models, as follows: (i) EGARCH is a model of the logarithm of the conditional variance, which implies that no restrictions on the parameters are required to ensure $h_{t}>0$; (ii) moment conditions are required for the GARCH and GJR models as they are dependent on lagged unconditional shocks, whereas EGARCH does not require moment conditions to be established as it depends on lagged conditional shocks (or standardized residuals); (iii) Shephard (1996) observed that $|\beta|<1$ is likely to be a sufficient condition for consistency of QMLE for EGARCH(1,1); (iv) as the standardized residuals appear in equation (7), $|\beta|<1$ would seem to be a sufficient condition for the existence of moments; and (v) in addition to being a sufficient condition for consistency, $|\beta|<1$ is also likely to be sufficient for asymptotic normality of the QMLE of $\operatorname{EGARCH}(1,1)$.

The three conditional volatility models given above are estimated under the following distributional assumptions on the conditional shocks: (1) Gaussian, (2) Student- $t$, with estimated degrees of freedom, and (3) Generalized Normal. As the models that incorporate the t distributed errors are estimated by QMLE, the resulting estimators are consistent and asymptotically normal, so they can be used for estimation, inference and forecasting.

\subsection{Exponentially Weighted Moving Average (EWMA)}

As an alternative to estimating the parameters of the appropriate conditional volatility models, Riskmetrics (1996) developed a model which estimates the conditional variances and covariances based on the exponentially weighted moving average (EWMA) method, which is, in effect, a restricted version of the $\operatorname{ARCH}(\infty)$ model. This symmetric approach forecasts the conditional variance at time $t$ as a linear combination of the lagged conditional variance and the squared unconditional shock at time $t-1$. The EWMA model calibrates the conditional variance as: 


$$
h_{t}=\lambda h_{t-1}+(1-\lambda) \varepsilon_{t-1}^{2}
$$

where $\lambda$ is a decay parameter. Riskmetrics (1996) suggests that $\lambda$ should be set at 0.94 for purposes of analysing daily data. As no parameters are estimated, there are no moment or logmoment conditions.

\subsection{Sequential VaR Combinations}

In this sub-section we consider the Bayesian approach to sequential forecast combination, as proposed in Billio et al. (2010), who combine optimally the forecast densities from different models that are estimated sequentially over time. Their method will be applied to the special case where the quantities to be combined are not forecast densities, but the quantiles (VaR) of different forecast densities.

Let $q_{t}$ be the recursive estimate of the $5 \%$ quantile of the empirical distribution of the subsample $r_{t-\tau+1}, \ldots r_{t}$, where $r_{t}$ denotes the S\&P500 returns, and the size of the rolling window is $\tau=100$. If $\mathrm{VaR}_{\mathrm{k}, \mathrm{t}}$ is the $\mathrm{VaR}$ forecast from model $k$, then $k=1,2$ and 3 for EGARCH, $\mathrm{EGARCH}_{\mathrm{G}}$ and $\mathrm{EGARCH}_{\mathrm{T}}$, respectively, $k=4,5$ and 6 for $\mathrm{GARCH}, \mathrm{GARCH}_{\mathrm{G}}$ and $\mathrm{GARCH}_{\mathrm{T}}$, respectively, and $k=7,8$ and 9 for $\mathrm{GJR}, \mathrm{GJR}_{\mathrm{G}}$ and $\mathrm{GJR}_{\mathrm{T}}$, respectively.

One possible way to account for the time-variations in the ability of the different models to forecast the quantiles is to combine the VaR forecasts linearly, as follows:

$$
q_{t}=\sum_{k=1}^{K} w_{k, t} V a R_{k, t}+\varepsilon_{t}, \quad \varepsilon_{t} \square N\left(0, \sigma_{\varepsilon}^{2}\right)
$$

where $\varepsilon_{t}$ is the residual forecasting error, which accounts for the fact that the true data generating process may not be within the set of models, and $w_{k, t}, k=1, \ldots, K$, is a set of weights to be determined. In order to obtain an almost everywhere bounded VaR measure, we consider a convex cobination scheme. We assume that the weights belong to the unit interval through the following multivariate transformation: 


$$
w_{k, t}=\exp \left(x_{k, t}\right)\left(\sum_{j=1}^{K} \exp \left(x_{j, t}\right)\right)^{-1}, k=1, \ldots, K
$$

where $x_{k, t}, k=1, \ldots, K$, is a set of real-valued latent variables.

The dynamics of the latent process, used in the combination scheme in equations (10)-(11), should be flexible enough to capture the time variations in forecast ability. In this section, we consider two alternative combination schemes that allow for randomness and time variations in the combination weights, as follows:

\section{(1) Random Walk (RW) Strategy}

$$
x_{k, t}=x_{k, t-1}+\eta_{k, t}, \eta_{k, t} \square N\left(0, \sigma_{\eta}^{2}\right)
$$

\section{(2) Charges-Driven (CD) Strategy}

$$
x_{k, t}=x_{k, t-1}-\left(H_{k, t}-H_{k, t-1}\right)+\eta_{k, t}, \eta_{k, t} \square N\left(0, \sigma_{\eta}^{2}\right)
$$

with $K=9$, where

$$
H_{k, t}=\sup \left\{-V a R_{k, t-1},-(3+g) \frac{1}{60} \sum_{p=1}^{60} V a R_{k, t-p}\right\}
$$

denotes daily capital charges at time $t$ for model $k, k=1, \ldots, K$. In the CD strategy, an increase in capital charges for a given model reduces its weight in the combination. The convexity constraints on the combination weights serve to emphasize the interpretation of the combination procedure as a measure of risk, which is bounded from above and below, as will be discussed below.

For the estimation of the combination model, we adopt a Bayesian approach. We consider the completed likelihood and estimate the hidden states (combination weights) of the model, assuming independent inverted gamma prior distributions for the scale parameters of the 
latent factor. Owing to the nonlinearity of the model, we use a nonlinear filtering approach for the joint estimation of both the parameters and the hidden states. Following Billio et al. (2010), we take the union of the parameter vector:

$$
\theta=\left(\sigma_{\varepsilon}^{2}, \sigma_{\eta}^{2}\right) \in \Theta
$$

where $\Theta=R_{+} \times R_{+}$and the state vector:

$$
x_{t}=\left(x_{1, t}, \ldots, x_{K, t}\right)^{\prime} \in \mathrm{X}
$$

where $\mathrm{X}=R^{K}$, and define the augmented state vector, $x_{t}=\left(x_{t}, \theta\right)^{\prime} \in \mathrm{Z}$, where $\mathrm{Z}=\mathrm{X} \times \Theta$.

Let $\tilde{q}_{t}=\left(\operatorname{VaR}_{1, t}, \ldots, V a R_{K, t}\right)^{\prime}$ be the vector of VaRs, and denote $u_{s: t}=\left(u_{s}, \ldots, u_{t}\right)^{\prime}$ as the collection of vectors from time 1 to $t$. The general state-space representation of the VaR combination scheme that is given by:

$$
\begin{gathered}
q_{t} \mid z_{t}, \tilde{q}_{t} \square p\left(q_{t} \mid z_{t}, \tilde{q}_{t}\right) \\
q_{t} \mid z_{t-1} \square p\left(z_{t} \mid z_{t-1}\right) \\
z_{0} \square p\left(z_{0}\right)
\end{gathered}
$$

can be used to define, respectively, the forecast and filtering densities:

$$
\begin{gathered}
p\left(z_{t} \mid q_{1: t-1}, \tilde{q}_{1: t-1}\right)=\int_{\mathrm{Z}} p\left(z_{t} \mid z_{t-1}\right) p\left(z_{t-1} \mid q_{1: t-1}, \tilde{q}_{1: t-1}\right) d z_{t-1} \\
p\left(z_{t} \mid q_{1: t}, \tilde{q}_{1: t}\right)=\frac{p\left(z_{t} \mid q_{1: t-1}, \tilde{q}_{1: t-1}\right) p\left(q_{t} \mid z_{t}, \tilde{q}_{t}\right)}{p\left(q_{t} \mid q_{1: t-1}, \tilde{q}_{1: t}\right)}
\end{gathered}
$$

The forecast and filtering densities cannot be solved analytically for the nonlinear combination model, which leads to the use of sequential Monte Carlo (SMC) techniques (see Doucet et al. (2001) for an introduction to SMC). We use the set of $N$ weighted random 
variables, $\Xi_{t}=\left\{z_{t-1}^{i}, \gamma_{t-1}^{i}\right\}_{i=1}^{N} \in Z^{N}$, called the particle set, to approximate the forecast and filtering densities with their empirical counterparts:

$$
\begin{gathered}
p_{N}\left(z_{t} \mid q_{1: t-1}, \tilde{q}_{1: t-1}\right)=\sum_{i=1}^{N} p\left(z_{t} \mid z_{t-1}, \tilde{q}_{1: t-1}\right) \gamma_{t-1}^{i} \delta_{\left\{z_{t-1}^{i}\right\}}\left(z_{t-1}\right) \\
p_{N}\left(z_{t} \mid q_{1: t}, \tilde{q}_{1: t}\right)=\sum_{i=1}^{N} \gamma_{t}^{i} \delta_{\left\{z_{t}^{i}\right\}}\left(z_{t}\right)
\end{gathered}
$$

respectively, where $\gamma_{t}^{i} \propto \gamma_{t-1}^{i} p\left(q_{t} \mid z_{t}^{i}, \tilde{q}_{t}\right)$ are importance weights, and $\delta_{\{x\}}(q)$ denotes the Dirac's mass centered at $x$. Note that the filtering density allows for the updating of the forecast combination weights on the basis of the past values of the $\mathrm{VaR}$ forecasts.

The empirical forecast density can be used to combine the VaRs, as follows:

$$
E\left(q_{t} \mid q_{1: t-1}, \tilde{q}_{1: t}\right) \approx \sum_{k=1}^{K} V a R_{k, t} \sum_{i=1}^{N} \gamma_{t}^{i} \frac{\exp \left(x_{k, t}^{i}\right)}{\sum_{j=1}^{K} \exp \left(x_{j, t}^{i}\right)}
$$

to obtain an expected $\mathrm{VaR}$, which is bounded from below and above for each observation by two VaRs in the following set:

$$
\min _{k=1, \ldots, 9}\left\{\operatorname{VaR}_{k, t}\right\} \leq E\left(q_{t} \mid q_{1: t-1}, \tilde{q}_{1: t}\right) \leq \max _{k=1, \ldots, 9}\left\{\operatorname{VaR}_{k, t}\right\}
$$

\section{Data}

As discussed in Chang et al. (2011), the data used in estimation and forecasting are closing daily prices (settlement prices) for the 30-day maturity CBOE VIX volatility index futures (ticker name VX), and were obtained from the Thomson Reuters-Data Stream Database for the period 26 March 2006 to 10 January 2011. The settlement price is calculated by the CBOE as the average of the closing bid and ask quote so as to reduce the noise due to any microstructure effects. The contracts are cash settled on the Wednesday 30 days prior to the 
third Friday on the calendar month immediately following the month in which the contract expires. The underlying asset is the VIX index that was originally introduced by Whaley (1993) as an index of implied volatility on the S\&P100. In 2003 the updated VIX was introduced based on the S\&P500 index.

VIX is a measure of the implied volatility of 30-day S\&P500 options. Its calculation is independent of an option pricing model and is calculated from the prices of the front month and next-to-front month S\&P500 at-the-money and out-the-money call and put options. The level of VIX represents a measure of the implied volatilities of the entire smile for a constant 30-day to maturity option chain. VIX is quoted in percentage points (for example, 30.0 VIX represents an implied volatility of $30.0 \%$ ). In order to invest in VIX, an investor can take a position in VIX futures or VIX options.

CBOE (2003) shows that VIX is a measure of the expected volatility of the S\&P500 over the next 30-days, with the prices of VIX futures being based on the current expectation of what the expected 30-day volatility will be at a particular time in the future (on the expiration date). Although the VIX futures should converge to the spot at expiration, it is possible to have significant disparities between the spot VIX and VIX futures prior to expiration. Figure 1 shows the daily VIX futures index together with the 30 day maturity VIX futures closing prices. VIX has a correlation (0.96) with the 30-day maturity VIX futures. Regarding volatility, VIX futures prices tend to show significantly lower volatility than VIX, which can be explained by the fact that VIX futures must be priced in a manner that reflects the mean reverting nature of VIX. For the whole sample, the standard deviation is 11.12 for VIX and 9.55 for VIX futures prices.

\section{[Insert Figure 1 here]}

In Figure 1 it can be seen that, from 2004 until 2007, the equity markets enjoyed a tranquil period, as did the VIX futures prices. After the first signs of a looming economic crisis, VIX futures rose sharply, in July 2007, after fluctuating around an average of 14. Following the Lehman Brothers collapse in September 2008, VIX futures appeared to jump to an all time high of over 66.23 in November 20, 2008. VIX futures prices returned to around \$20 in 2009, but with the Greek crisis in May 2010, the VIX futures prices again jumped to around \$36. 
If $P_{t}$ denotes the closing prices of the VIX futures contract at time $t$, the returns at time $t\left(R_{t}\right)$ are defined as:

$$
R_{t}=100 * \log \left(P_{t} / P_{t-1}\right)
$$

Figure 2 shows the daily VIX futures returns, and the descriptive statistics for the daily returns are given in Table 2. The returns to the VIX futures are driven by changes in expectations of implied volatility. Figure 3 shows the histograms for the daily returns, together with the theoretical Gaussian and Student-t probability density functions and a kernel density estimator. The Student-t density fits the returns distributions better than does its Gaussian counterpart.

\section{[Insert Figures 2-4 and Tables 2-4 here]}

It is also interesting to examine the returns distributions for the three periods relating to before the GFC (1 January, 2008 - 11 August, 2008), during the GFC (12 August, 2008-9 March, 2009), and after the GFC (10 March, 2009 - 10 January, 2011). We choose these dates because they coincide with the peaks and troughs of the S\&P500, as we do in previous papers, such as McAleer et al. (2010b, c). As can be seen in Figure 4, there are changes in the shapes of the underlying probability density functions before, during and after the crisis. We graph the empirical distributions, together with the Normal, Student-t and a kernel density estimator, for the three periods. Clearly, the shape of the densities changes from one period to another. It is worth noting that before the crisis, there are some very large positive returns around +17 , while during the crisis there are many large negative returns with values around -20. However after the crisis, we again have some large, but not as large as before, positive returns with values around +8 . This illustrates, together with the data on skewness and kurtosis in Tables 3 and 4 , that the distributions are different across time as is.

Table 3 shows tests for equality of means and variances across the three periods. All of them

clearly reject equality. When we test the null hypothesis of equality of distributions using the Kolmogorov-Smirnov test, for the periods before and during the crisis, we have only a marginal rejection, while we reject equality for the periods during and after the crisis. 
Table 4 - panel a gives the descriptive statistics for VIX futures, which suggest heterogeneity in the empirical distributions. As seen in Table 4 - panel b, all the empirical distributions are positively skewed (0.88) and leptokurtic (8.21). The before GFC period exhibits the highest skewness (0.93), followed by the after GFC period (0.86), and with the during GFC period showing negative skewness (-0.15). The maximum and minimum (in parentheses) for the before, during and after GFC periods are, respectively, $16.25(-8.55), 13.23(-19.93)$ and 13.40 (-13.46), respectively. The returns for the three periods before, during and after the GFC show high kurtosis of 6.01, 4.25 and 4.90, respectively. The total period has the highest value kurtosis of 8.21 .

Regarding the returns volatility, several measures of volatility are available in the literature. In order to gain some intuition, we adopt the measure proposed in Franses and van Dijk (1999), wherein the true volatility of returns is defined as:

$$
V_{t}=\left(R_{t}-E\left(R_{t} \mid F_{t-1}\right)\right)^{2}
$$

\section{[Insert Figure 5 here]}

where $F_{t-1}$ is the information set at time t-1. Figure 5 presents the square root of $V_{t}$ in equation Error! Reference source not found. as “volatilities”. The series exhibit clustering that should captured by an appropriate time series model. Until January 2007, a month before the first reports of subprime losses, the volatility of the series seems to be stable. The volatility reached an all time peak on February 27, 2007, when it climbed to 0.26 (the median for the entire sample is 0.023 ), as the US equity market had its worst day in four years. Then it remained above historic levels, but the VIX futures volatility increases again after August 2008, due in large part to the worsening global credit environment, with a maximum again on November 3, 2008. Then the volatility remained low until the news about the sovereign debt crisis in the Euro zone created another spike in volatility in the first week of May, when the VIX futures reached 35 with a high volatility in returns.

\section{VaR of VIX and Evaluation Framework}


As discussed in McAleer et al. (2010c), the GFC has affected the optimal risk management strategies by changing the best model for minimizing daily capital charges in all the cases analyzed. The purpose of this section is to provide an analysis of risk management strategies when considering 30-day maturity VIX futures before, during and after the GFC, using nonBayesian and Bayesian methods.

As ADIs need not restrict themselves to using only a single risk model, McAleer et al. (2010b) proposed a risk management strategy that uses combinations of several models for forecasting VaR. It was found that an aggressive risk management strategy (namely, choosing the Supremum of VaR forecasts, or an upperbound) yielded the lowest mean capital charges and largest number of violations. On the other hand, a conservative risk management strategy (namely, by choosing the Infinum, or lowerbound) had far fewer violations, and correspondingly higher mean daily capital charges.

McAleer et al. (2010c) forecast VaR using ten single GARCH-type models with different error distributions. Additionally, they analyzed twelve new strategies based on combinations of the previous standard single-model forecasts of VaR, namely: Infinum (0th percentile), Supremum (100th percentile), Average, Median and nine additional strategies based on the $10^{\text {th }}$ through to the $90^{\text {th }}$ percentiles. This is intended to select a robust VaR forecast, irrespective of the time period, that provides reasonable daily capital charges and number of violation penalties under the Basel Accord. They found that the Median ( $50^{\text {th }}$ percentile) is a GFC-robust strategy, in the sense that maintaining the same risk management strategy before, during and after the GFC leads to comparatively low daily capital charges and violation penalties under the Basel Accord.

Chang et al. (2011) conducted a similar exercise to analyze the risk management performance of existing VaR forecasting models, as permitted under the Basel II framework, when applied to VIX futures prices. The following section extends this work by using Bayesian methods that combine individual models optimally.

\subsection{Evaluating Non-Bayesian Risk Management Strategies}

Based on the S\&P500 peaks and troughs, before the GFC is prior to 11 August 2008, during the GFC is from 12 August 2008 through to 9 March 2009, and after the GFC is from 10 
March 2009 onwards. Chang et al. (2011) discussed the criteria used for comparison of the strategies for forecasting the volatility and VaR of the VIX futures returns, namely average daily capital charges (AvDCC), normalized number of violations, (NoV), failure rate (FailRa), accumulated losses, (AcLoss), and asymmetric linear tick loss function (AlTick).

The basic criterion for choosing a strategy is minimizing the average daily capital charges subject to the constraint that the normalized number of violations (equivalently, the percentage of violations) is within the limits allowed under the Basel II Accord. This criterion was used because it is assumed that ADIs want to maximize profits, and hence minimize costs. One of the relevant costs of the ADIs is the daily capital charges which they incur for holding their capital requirements (see also McAleer et al. (2010a)).

Chang et al. (2011) considered the accumulated losses, which are not taken into account in the rules of Basel II, but which might be considered in the future. In principle, low values of this criterion are desirable. We also consider the asymmetric loss tick function, which should be minimized. These criteria are also used in the literature and here we find that when we are choosing an optimal strategy they tend to agree with our primary criterion (see also McAleer et al. (2010c)).

The main conclusions in Chang et al. (2011) are as follows:

1. Before the GFC, the best strategy for minimizing daily capital charges (DCC) is the Supremum. It has the lowest AvDCC and the highest number of violations, but is still within the limits of the Basel II Accord. The Supremum also has the next to lowest asymmetric linear tick loss function, but also the highest accumulated losses. The Supremum is clearly the best strategy for forecasting the VaR of VIX futures before the GFC.

2. During the GFC, the Supremum has the lowest average daily capital charges and highest (but admissible) number of violations. Moreover, it has one of the three lowest values of AlTick values. However, the Supremum shows the highest accumulated losses of all the models. In general, the Supremum is the optimal strategy for managing risk under the Basel II Accord during the GFC. 
3. After the GFC, the Supremum has the lowest average daily capital charges, at the cost of the highest (but admissible) number of violations. Moreover, the Supremum has one of the three lowest asymmetric linear tick loss function values, at the expense of the highest accumulated losses.

The Supremum emerges as the optimal strategy for minimizing daily capital charges for VIX futures, at the expense of the number of violations, and accumulated losses which are permissible under the Basel II Accord. This suggests that there is still room for improving the risk management within the limits of Basel II by using the Supremum strategy.

A comparison with a leading competitor, Riskmetrics showed that the Supremum consistently dominated Riskmetrics. The Supremum always hds lower daily capital charges, with the same number of violations, before, during and after the GFC. The Supremum is a strategy which is riskier than the others considered, indicating that the others may be too conservative. This may arise from the extra flexibility afforded by being able to switch between risk models.

In summary, Chang et al. (2011) found the Supremum to be the risk management strategy that performed the best across all the considered strategies and time periods. It was also a GFCrobust strategy, as defined in McAleer et al. (2010c, 2011), meaning it is an optimal strategy that is valid before, during and after the GFC.

\subsection{Evaluating Bayesian Risk Management Strategies}

The VaR forecasts obtained from the combination of the time-varying VaRs is usually close to the median of the combined $\mathrm{VaR}$, even if, as in some periods of high volatility, the combined $\mathrm{VaR}$ tends to be higher than the median (see Figure 6). In the same figure, we also show the upper, $\max _{k=1, \ldots, 9}\left\{\operatorname{VaR}_{k, t}\right\}$, and lower, $\min _{k=1, \ldots, 9}\left\{\operatorname{VaR}_{k, t}\right\}$, bounds for the combined VaR, which give an indication of the average variation of the combined VaR.

In order to investigate the features of the two combination strategies, RW and CD, Figure 7 shows the differences between the median strategy, on the one hand, and the RW and CD strategies, on the other. In periods of low volatility, the combination strategies, RW and CD, have lower VaRs than the median (given as positive values in Figure 2), while in periods of 
high volatility, RW and CD have higher VaRs than the median (given as negative values in Figure 2). It is also worth noting that the CD strategy during the GFC is more conservative than the RW strategy, exhibiting a higher VaR than the median and RW.

\section{[Insert Figures 6-7 here]}

Similar results are evident in Table 6, in which we evaluate the sensitivity of the alternative strategies to recursive estimation of the quantiles. We evaluate the performance in terms of mean daily capital charges for different choices of the rolling windows size, $\tau=10,50,100$, and find no substantial differences between the results with different window sizes during the periods before and after the GFC. On the contrary, as might be expected, the empirical results are more sensitive to the choice of window size, that is, to the estimation of the quantiles, during the GFC.

\section{[Insert Table 6 here]}

In Figure 8 we show the weights of the combination strategies that are assigned to the three different groups of models. The sum of the weights of the three models in the EGARCH class is considerably higher than for the three models in each of the GARCH and GJR classes. Moreover, the sum is quite stable over time, especially for RW, and contributes around 75$80 \%$ to the model combination on the basis of both RW and CD. The three GJR models contribute a higher sum (around 10-15\%) to the VaR forecasts than do the three GARCH models (at 10\%, on average), although after the GFC (from 1 August 2008 to 1 April 2009) the difference between the GJR and GARCH models is significantly smaller.

Given the relative contributions in terms of predictive performance of the three model classes to the sum, it is useful to investigate the predictive performance of the different models within each model class (see Figures 9-11). Within the EGARCH model class in Figure 9, for the RW strategy, $\mathrm{EGARCH}_{\mathrm{G}}$ underperforms badly relative to the other two models. The difference between EGARCH and $\mathrm{EGARCH}_{\mathrm{T}}$ is small before the GFC, is reduced in the second half of the GFC, and increases substantially thereafter. In particular, the importance of EGARCH in this class increases until May 2010. Overall, the predictive performance of EGARCH is generally high, first improving then deteriorating slightly relative to $\mathrm{EGARCH}_{\mathrm{T}}$ after the GFC. The predictive performance of $\mathrm{EGARCH}_{\mathrm{T}}$ deteriorates throughout the samle, 
but improves eell after the GFC, starting in May 2010. For the CD strategy, EGARCH dominates before the GFC, but EGARCH dominates during and after the GFC.

\section{[Insert Figures 8-11 here]}

In the GARCH class of models in Figure 10, $\mathrm{GARCH}_{\mathrm{G}}$ usually has high predictive performance, which increases in periods of high volatility and sudden changes in the series (especially between 1 August 2008 and 1 April 2009), and decreases during periods of lower volatility, such as well after the GFC. An interesting result arises from a comparison of GARCH and $\mathrm{GARCH}_{\mathrm{T}}$. In the combination forecasting model, GARCH exhibits increasing weights and, after the GFC, the weights of GARCH become higher than those associated with $\mathrm{GARCH}_{\mathrm{T}}$.

A similar phenomenon can be seen in Figure 11 for the Student-t version of the GJR model, $\mathrm{GJR}_{\mathrm{T}}$, which usually underperforms relative to the other two models, and which exhibits increasing differences with respect to $\mathrm{GJR}_{\mathrm{G}}$ and GJR after the GFC. It is interesting to observe the relation between GJR and $\mathrm{GJR}_{\mathrm{G}}$, with $\mathrm{GJR}_{\mathrm{G}}$ usually having a greater weight than GJR, but with a reversal observed during the middle part of the GFC. In particular, GJR had greater weights than $\mathrm{GJR}_{\mathrm{G}}$ in the combination model in 2009 ..

\section{Conclusion}

In the spectrum of financial assets, VIX futures prices are a relatively new financial product. As with any financial asset, VIX futures are subject to risk. In this paper we analyzed the performance of a variety of strategies for managing the risk, through forecasting VaR, of VIX futures under the Basel II Accord, before, during and after the global financial crisis (GFC) of 2008-09.

We forecast VaR using well known univariate model strategies, as well as new strategies based on combinations of risk models, that were proposed and analyzed in McAleer et al. (2009, 2010c, 2011). 
The candidate strategies for forecasting VaR of the VIX futures, and for managing risk under the Basel II Accord, were several univariate models, such as Riskmetrics, GARCH, EGARCH and GJR, each subject to different error distributions. We also used several more sophisticated strategies that combined single models, such as the Supremum, Infinum, Average, Median and the $10^{\text {th }}$ through $90^{\text {th }}$ percentiles of the point values of the forecasts of the univariate models.

Our main criterion for choosing between strategies is minimizing the average daily capital charges subject to the constraint that the number of violations (equivalently, the percentage of violations) is within the limits allowed by the Basel II Accord. Additionally, we consider the accumulated losses and asymmetric loss tick function, each of which would desirably have low values.

The principal empirical conclusions of the paper for non-Bayesian risk management strategies can be summarized as follows:

1. Before the GFC, the Supremum has the lowest Average Daily Capital Charges (AvDCC) and the highest (though admissible) number of violations under the Basel II Accord.

2. During the GFC, the Supremum has the lowest AvDCC and highest (but admissible) number of violations.

3. After the GFC, the Supremum has the lowest AvDCC, at the expense of the highest (but admissible) number of violations.

The Supremum dominates Riskmetrics consistently as it always has lower daily capital charges, with the same number of violations across all time periods: before, during and after the GFC. The Supremum, in our case, is a strategy which is more risky than the individual models considered, indicating that they may be too conservative, for minimizing daily capital charges.

The attraction for risk managers in using the Supremum strategy for this asset is that they do not need to keep changing the rules for generating daily VaR forecasts. The Supremum is an aggressive and profitable risk strategy for calculating VaR forecasts for VIX futures, both in 
tranquil and in turbulent times. However, the Supremum is not always the best strategy for all assets and all periods, as illustrated in McAleer et al. (2010c, 2011) and Chang et al. (2011).

For Bayesian risk management strategies, the principal empirical conclusions of the paper are quite clear. The sequential analysis of the VaR forecasting performance of the different models are useful in understanding which class of the three models, and which model within each class, should be used in different stages before, during and after the GFC, to estimate risk and forecast VaR. Moreover, the use of time-varying weights permits a dynamic combination of the different models across three classes to obtain a more accurate VaR forecasts than the estimates and forecasts that might be produced by a single model of risk.

The idea of combining different VaR forecasting models is entirely within the spirit of the Basel Accord, although its use would require approval by the regulatory authorities, as for any forecasting model. This approach is not at all computationally demanding, even though several models need to be specified and estimated over time. 


\section{References}

Basel Committee on Banking Supervision, (1988), International Convergence of Capital Measurement and Capital Standards, BIS, Basel, Switzerland.

Basel Committee on Banking Supervision, (1995), An Internal Model-Based Approach to Market Risk Capital Requirements, BIS, Basel, Switzerland.

Basel Committee on Banking Supervision, (1996), Supervisory Framework for the Use of "Backtesting” in Conjunction with the Internal Model-Based Approach to Market Risk Capital Requirements, BIS, Basel, Switzerland.

Basel Committee on Banking Supervision, (2006), International Convergence of Capital Measurement and Capital Standards, A Revised Framework Comprehensive Version, BIS, Basel, Switzerland.

Berkowitz, J. and J. O’Brien (2001), How accurate are value-at-risk models at commercial banks?, Discussion Paper, Federal Reserve Board.

Billio, M., R. Casarin, F. Ravazzolo and H.K. van Dijk (2010), Combining predictive densities using Bayesian filtering with applications to US economics data, Norges Bank Working Paper No.2010/29.

Black, F. (1976), Studies of stock market volatility changes, in 1976 Proceedings of the American Statistical Association, Business \& Economic Statistics Section, pp. 177-181.

Bollerslev, T. (1986), Generalised autoregressive conditional heteroscedasticity, Journal of Econometrics, 31, 307-327.

Borio, C. (2008), The financial turmoil of 2007-?: A preliminary assessment and some policy considerations, BIS Working Papers No 251, Bank for International Settlements, Basel, Switzerland.

Caporin, M. and M. McAleer (2010a), The Ten Commandments for managing investments, Journal of Economic Surveys, 24, 196-200.

Caporin, M. and M. McAleer (2010b), Model selection and testing of conditional and stochastic volatility models, to appear in L. Bauwens, C. Hafner and S. Laurent (eds.), Handbook on Financial Engineering and Econometrics: Volatility Models and Their Applications, Wiley, New York (Available at SSRN: http://ssrn.com/abstract=1676826).

Chang, C.-L., J.-A. Jimenez-Martin, M. McAleer and T. Perez Amaral (2011), Risk management of risk under the Basel Accord: Forecasting value-at-risk of VIX futures, to appear in Managerial Finance (Available at SSRN: http://ssrn.com/abstract=1765202). 
Chicago Board Options Exchange (2003), VIX: CBOE volatility index, Working paper, Chicago.

Doucet, A., J.G. Freitas and J. Gordon (2001), Sequential Monte Carlo Methods in Practice, Springer Verlag, New York.

Engle, R.F. (1982), Autoregressive conditional heteroscedasticity with estimates of the variance of United Kingdom inflation, Econometrica, 50, 987-1007.

Franses, P.H. and D. van Dijk (1999), Nonlinear Time Series Models in Empirical Finance, Cambridge, Cambridge University Press.

Gizycki, M. and N. Hereford (1998), Assessing the dispersion in banks' estimates of market risk: the results of a value-at-risk survey, Discussion Paper 1, Australian Prudential Regulation Authority.

Glosten, L., R. Jagannathan and D. Runkle (1992), On the relation between the expected value and volatility of nominal excess return on stocks, Journal of Finance, 46, 17791801.

Huskaj, B. (2009), A value-at-risk analysis of VIX futures long memory, heavy tails, and asymmetry. Available at SSRN: http://ssrn.com/abstract=1495229.

Jimenez-Martin, J.-A., McAleer, M. and T. Pérez-Amaral (2009), The Ten Commandments for managing value-at-risk under the Basel II Accord, Journal of Economic Surveys, 23, 850-855.

Jorion, P. (2000), Value at Risk: The New Benchmark for Managing Financial Risk, McGraw-Hill, New York.

Li, W.K., S. Ling and M. McAleer (2002), Recent theoretical results for time series models with GARCH errors, Journal of Economic Surveys, 16, 245-269. Reprinted in M. McAleer and L. Oxley (eds.), Contributions to Financial Econometrics: Theoretical and Practical Issues, Blackwell, Oxford, 2002, pp. 9-33.

Ling, S. and M. McAleer (2002a), Stationarity and the existence of moments of a family of GARCH processes, Journal of Econometrics, 106, 109-117.

Ling, S. and M. McAleer (2002b), Necessary and sufficient moment conditions for the GARCH(r,s) and asymmetric power GARCH(r,s) models, Econometric Theory, 18, 722-729.

Ling, S. and M. McAleer, (2003a), Asymptotic theory for a vector ARMA-GARCH model, Econometric Theory, 19, 278-308.

Ling, S. and M. McAleer (2003b), On adaptive estimation in nonstationary ARMA models with GARCH errors, Annals of Statistics, 31, 642-674. 
Lopez, J.A. (1999), Methods for evaluating value-at-risk estimates, Economic Review, Federal Reserve Bank of San Francisco, pp. 3-17.

McAleer, M. (2005), Automated inference and learning in modeling financial volatility, Econometric Theory, 21, 232-261.

McAleer, M. (2009), The Ten Commandments for optimizing value-at-risk and daily capital charges, Journal of Economic Surveys, 23, 831-849.

McAleer, M., F. Chan and D. Marinova (2007), An econometric analysis of asymmetric volatility: theory and application to patents, Journal of Econometrics, 139, 259-284.

McAleer, M., J.-Á. Jiménez-Martin and T. Pérez-Amaral (2010a), A decision rule to minimize daily capital charges in forecasting value-at-risk, Journal of Forecasting, 29, 617-634.

McAleer, M., J.-Á. Jiménez-Martin and T. Pérez-Amaral (2010b), Has the Basel II Accord encouraged risk management during the 2008-09 financial crisis?, Available at SSRN: http://ssrn.com/abstract=1397239.

McAleer, M., J.-Á. Jiménez-Martin and T. Pérez-Amaral (2010c), GFC-robust risk management strategies under the Basel Accord, Available at SSRN: http://ssrn.com/abstract=1688385.

McAleer, M., J.-Á. Jiménez-Martin and T. Pérez-Amaral (2011) International evidence on GFC-robust forecasts for risk management under the Basel Accord. Available at SSRN: http://ssrn.com/abstract=1741565.

McAleer, M. and B. da Veiga (2008a), Forecasting value-at-risk with a parsimonious portfolio spillover GARCH (PS-GARCH) model, Journal of Forecasting, 27, 1-19.

McAleer, M. and B. da Veiga (2008b), Single index and portfolio models for forecasting value-at-risk thresholds, Journal of Forecasting, 27, 217-235.

McAleer, M. and C. Wiphatthanananthakul (2010), A simple expected volatility (SEV) index: Application to SET50 index options, Mathematics and Computers in Simulation, 80, 2079-2090.

Nelson, D.B. (1991), Conditional heteroscedasticity in asset returns: A new approach, Econometrica, 59, 347-370.

Pérignon, C., Z.-Y. Deng and Z.-J. Wang (2008), Do banks overstate their value-at-risk?, Journal of Banking \& Finance, 32, 783-794.

Riskmetrics (1996), J.P. Morgan Technical Document, $4^{\text {th }}$ Edition, New York, J.P. Morgan. 
Shephard, N. (1996), Statistical aspects of ARCH and stochastic volatility, in O.E. BarndorffNielsen, D.R. Cox and D.V. Hinkley (eds.), Statistical Models in Econometrics, Finance and Other Fields, Chapman \& Hall, London, 1-67.

Stahl, G. (1997), Three cheers, Risk, 10, pp. 67-69.

Whaley, RE., 1993, Derivatives on market volatility: Hedging tools long overdue, Journal of Derivatives, 1, 71-84.

Zumbach, G. (2007), A Gentle Introduction to the RM 2006 Methodology, New York, Riskmetrics Group. 
Figure 1

VIX and 30-day Maturity VIX Futures Closing Prices

26 March 2004 - 10 January 2011

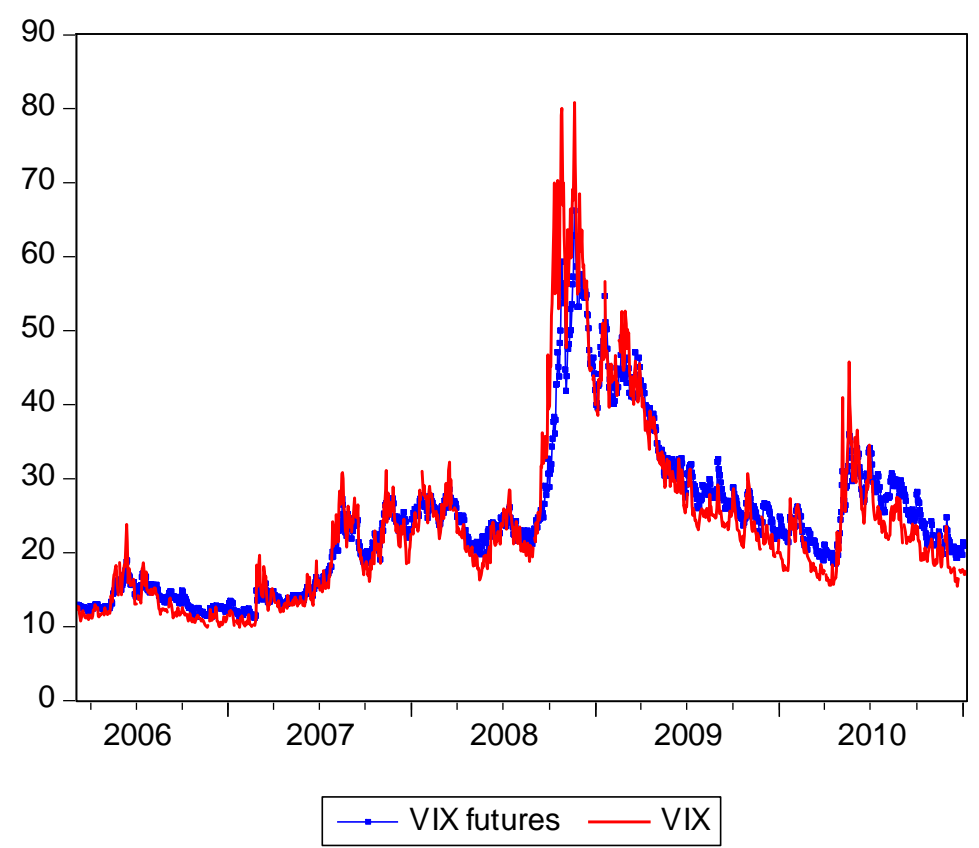


Figure 2

30-day Maturity VIX Futures Returns

26 March 2004 - 10 January 2011

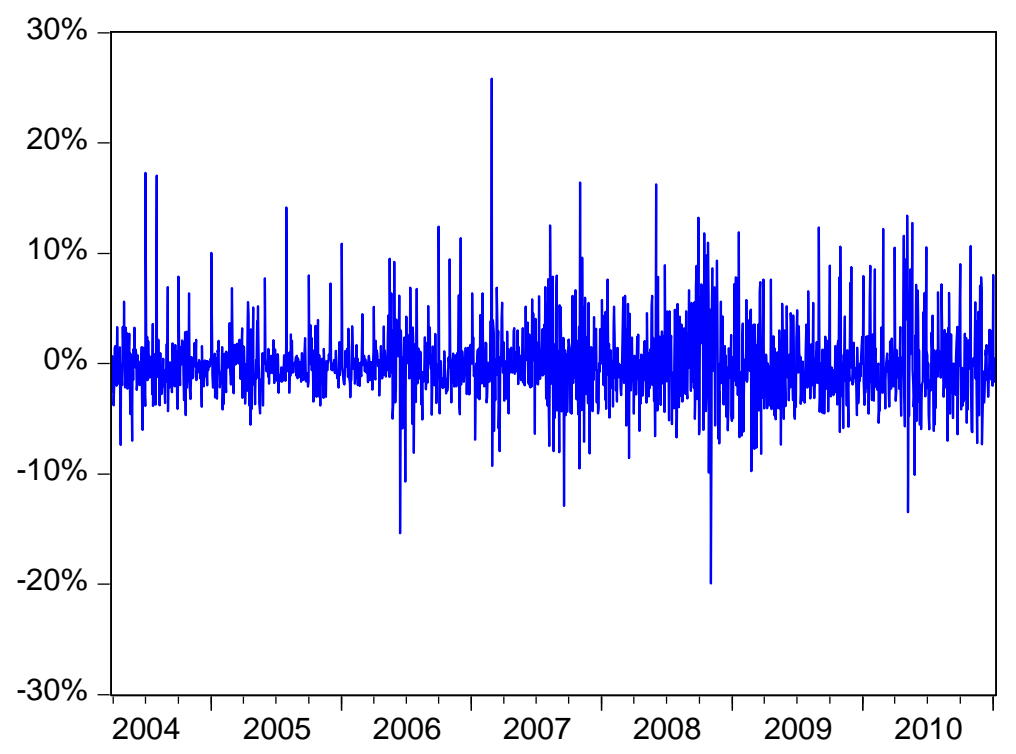


Figure 3

Histogram, Normal and Student-t Distributions and Kernel Density Estimator for 30-day Maturity VIX Futures Returns

26 March 2006 - 10 January 2011

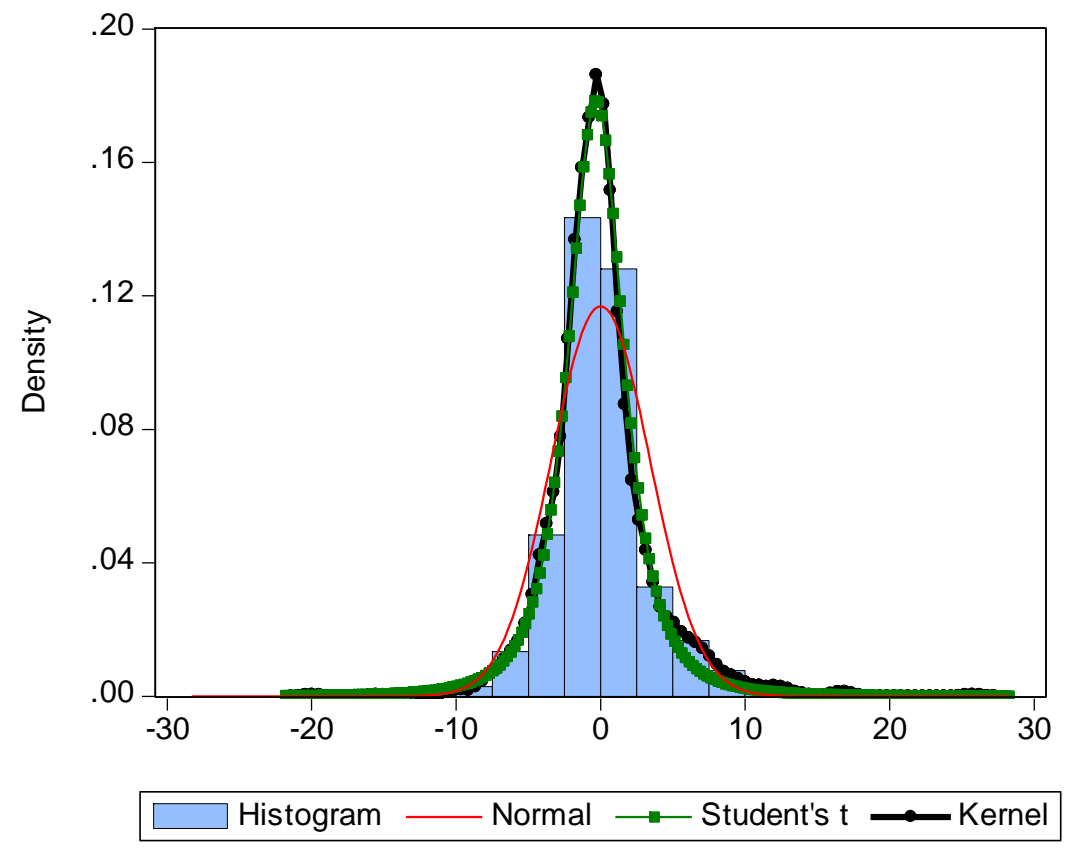


Figure 4

30-day Maturity VIX Futures Returns

Histogram, Normal, Student-t and Kernel Density Estimator

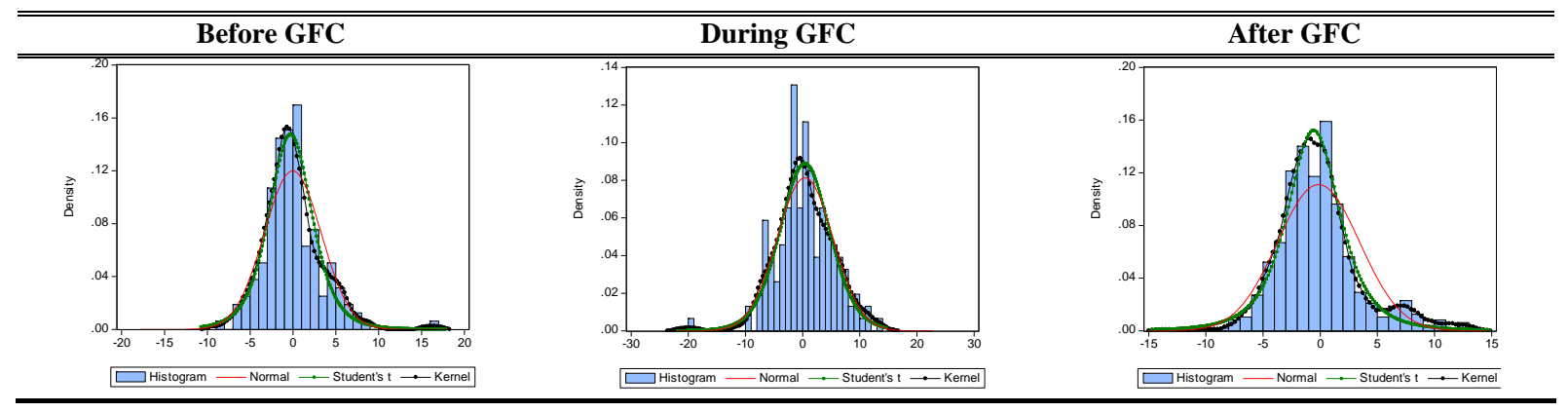


Figure 5

Volatility of 30-day Maturity VIX Futures Returns

26 March 2004 - 10 January 2011

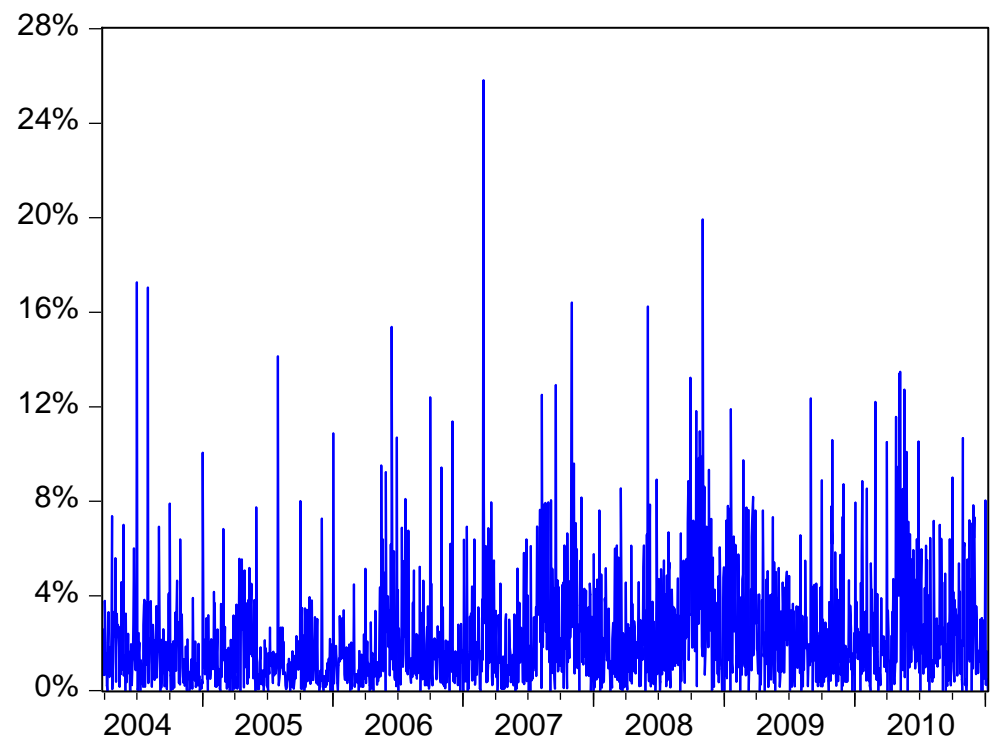



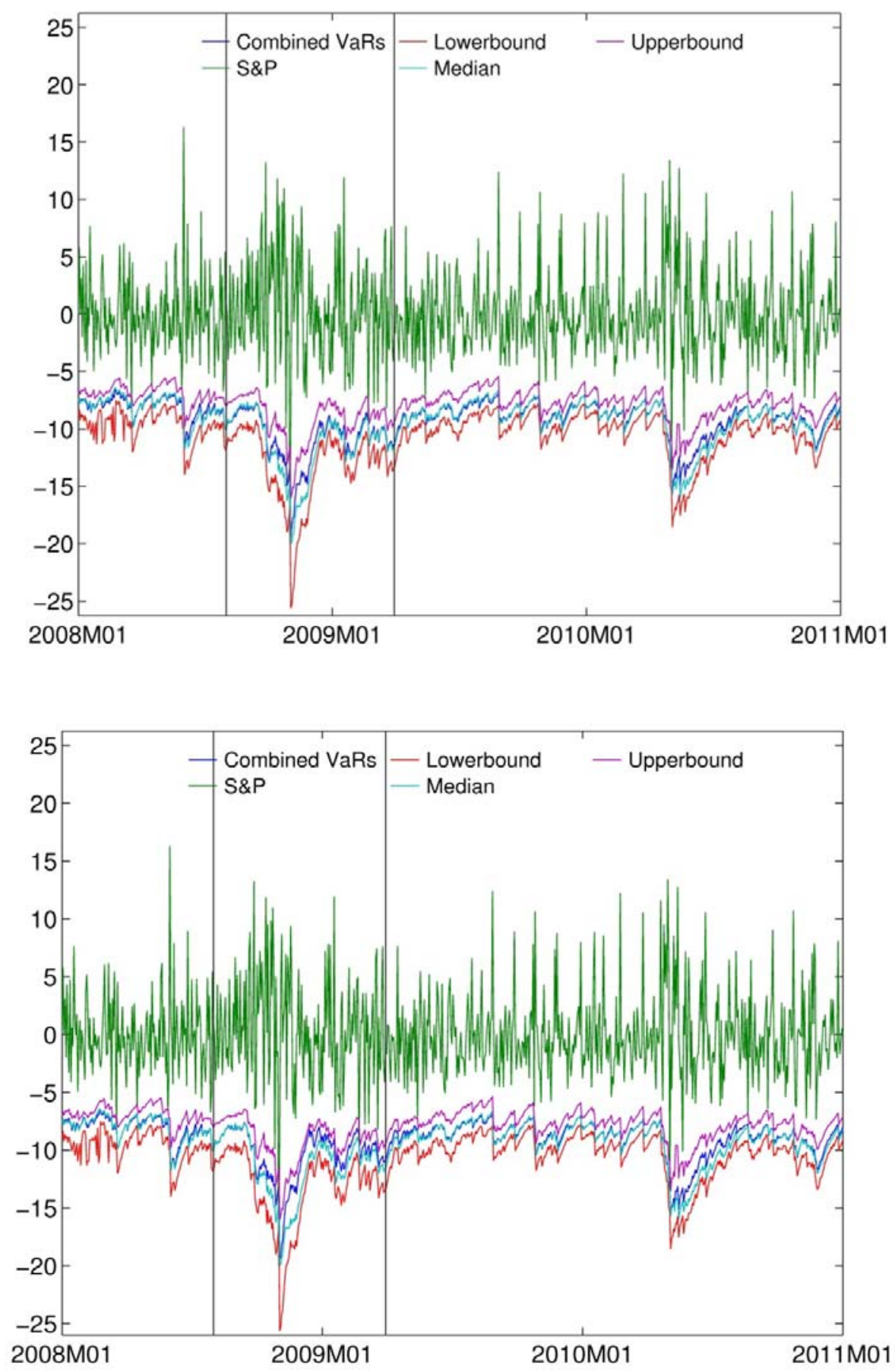

Figure 6

RW (upper) and CD (lower) strategies. The S\&P500 index log-returns (gray line) are shown with the median VaR (light blue) and combined VaR (dark blue), with the upper and lower bounds of VaR. The vertical lines correspond to the possible starting (1 August 2008) and finishing (1 April 2009) dates of the GFC. 


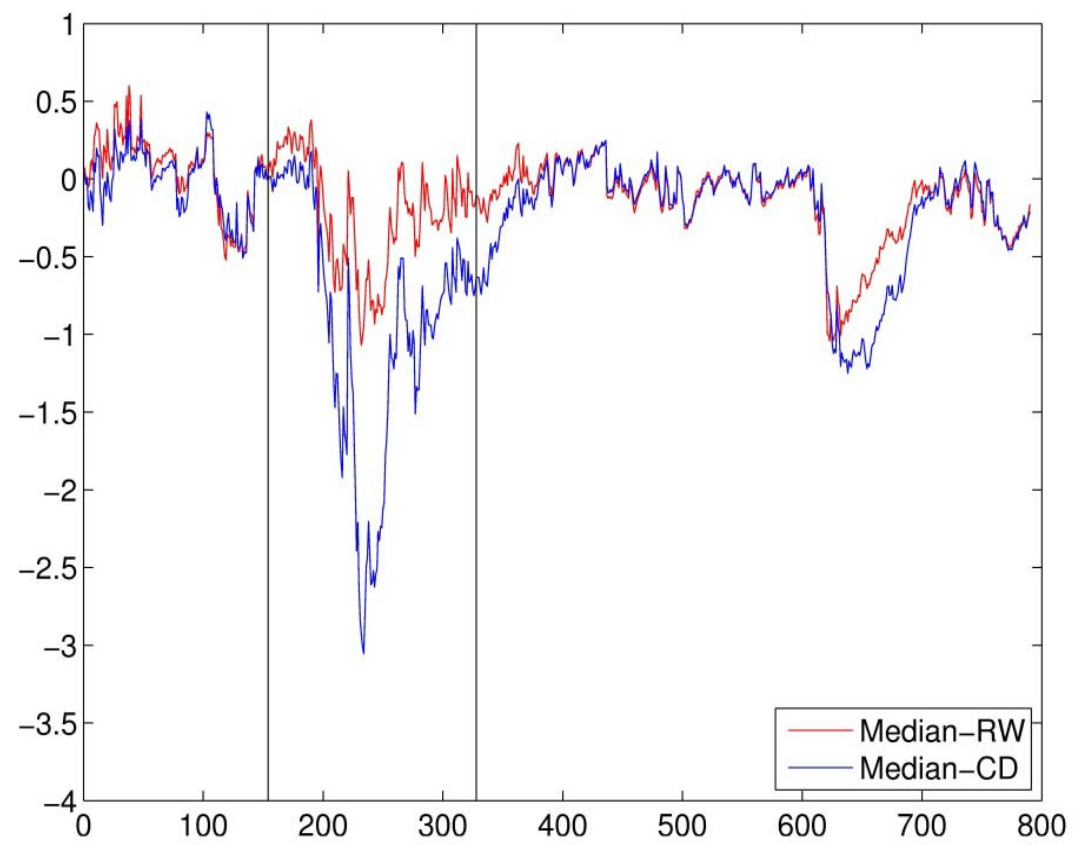

Figure 7

Differences between VaRs generated by the median, RW and CD strategies. 

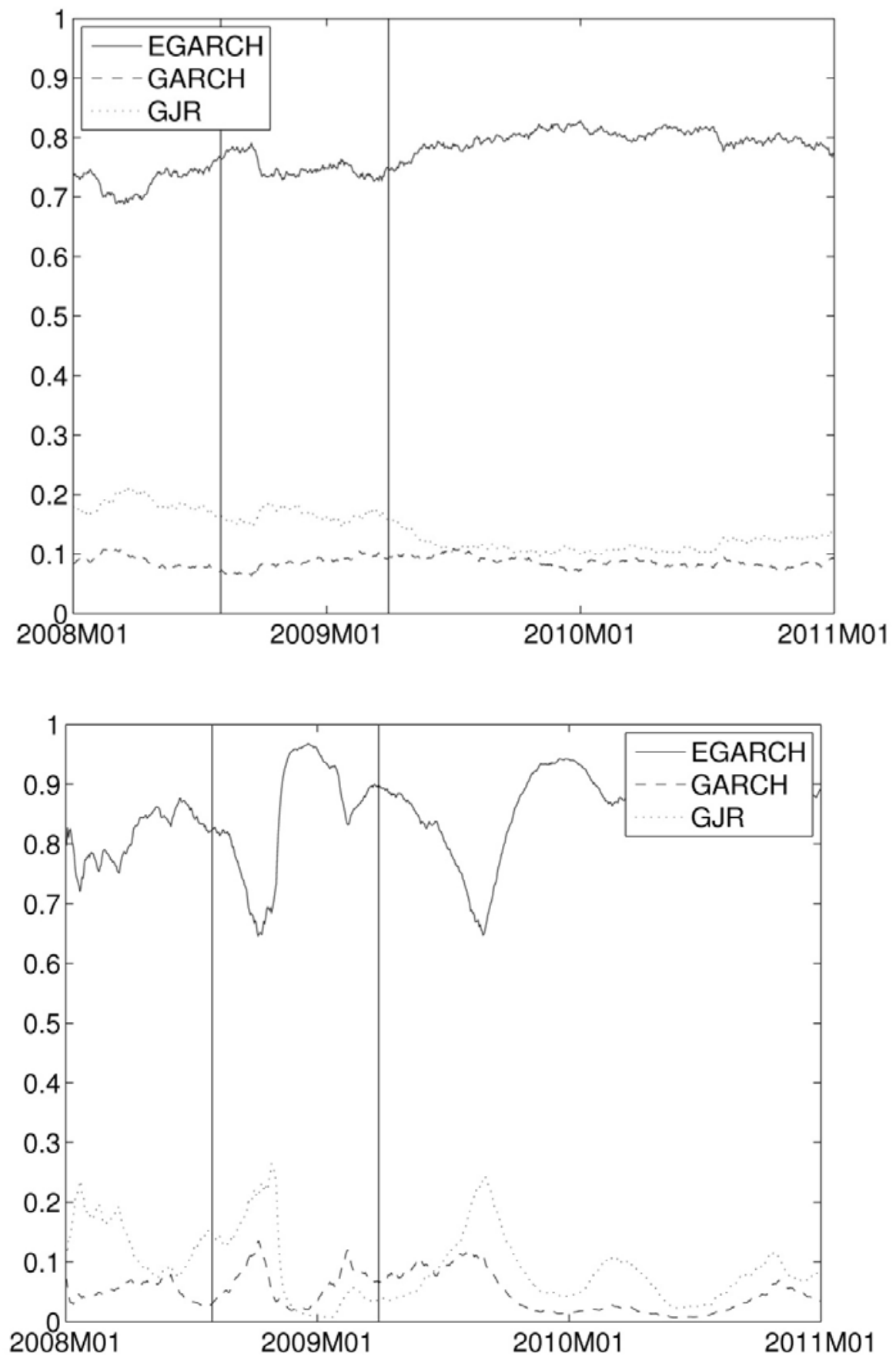

Figure 8

RW (upper) and CD (lower) strategies using filtered combination weights for three different classes of EGARCH, GARCH and GJR models. The vertical lines correspond to the possible starting (1 August 2008) and finishing (1 April 2009) dates of the GFC. 

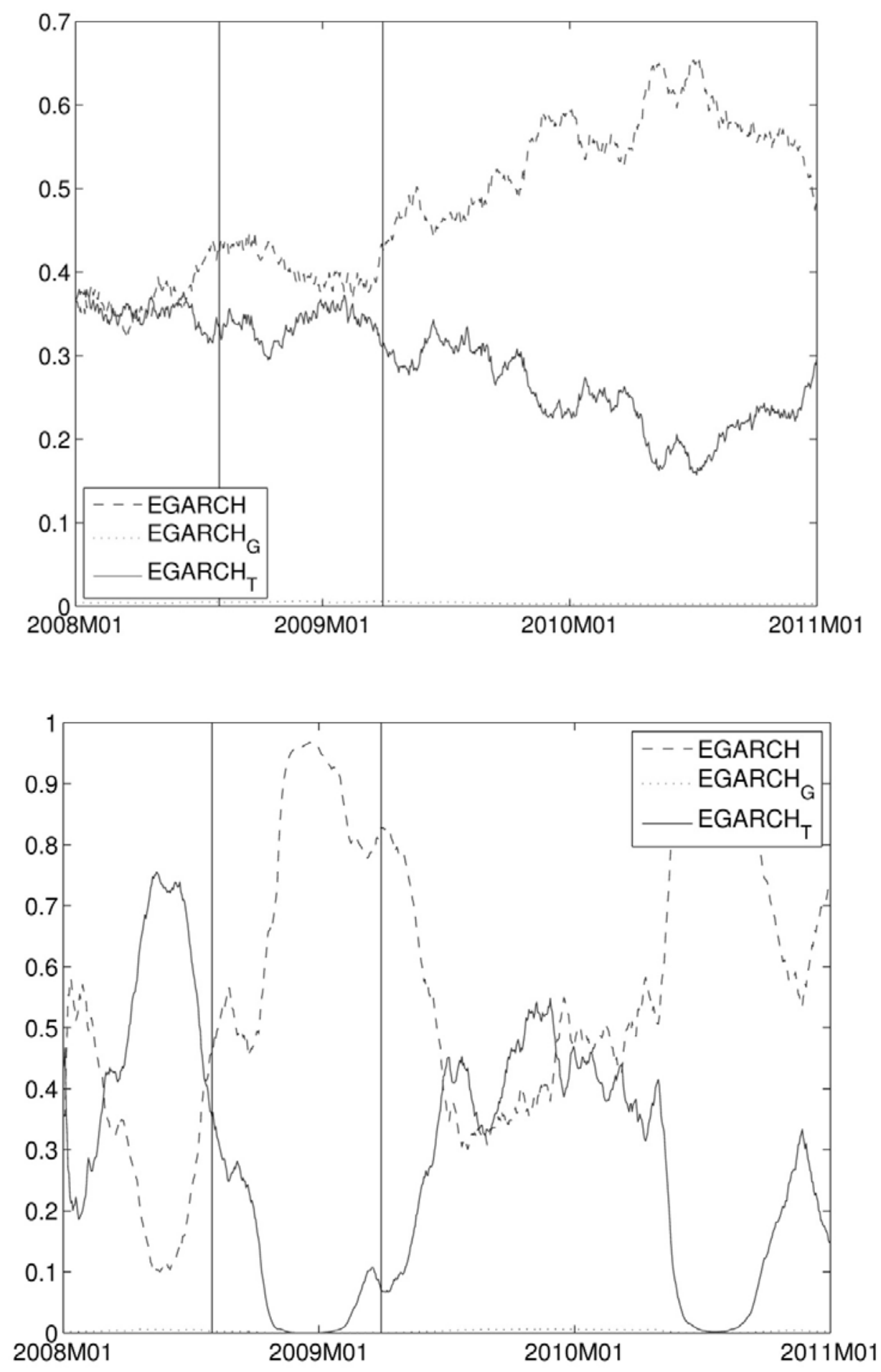

Figure 9

RW (upper) and CD (lower) strategies using filtered combination weights for three different EGARCH models. The vertical lines correspond to the possible starting (1 August 2008) and finishing (1 April 2009) dates of the GFC. 

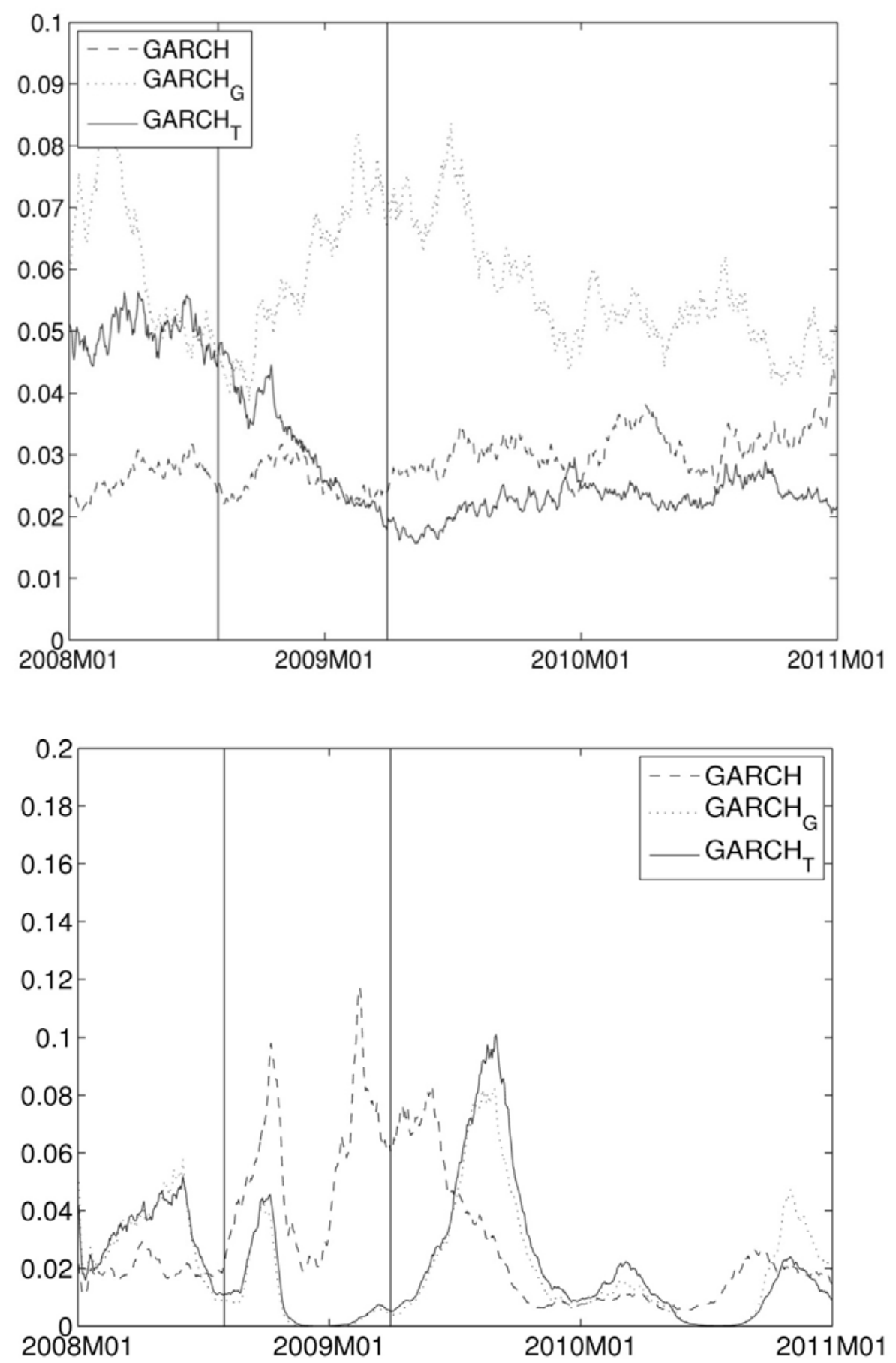

Figure 10

RW (upper) and CD (lower) strategies using filtered combination weights for three different GARCH models. The vertical lines correspond to the possible starting (1 August 2008) and finishing (1 April 2009) dates of the GFC. 

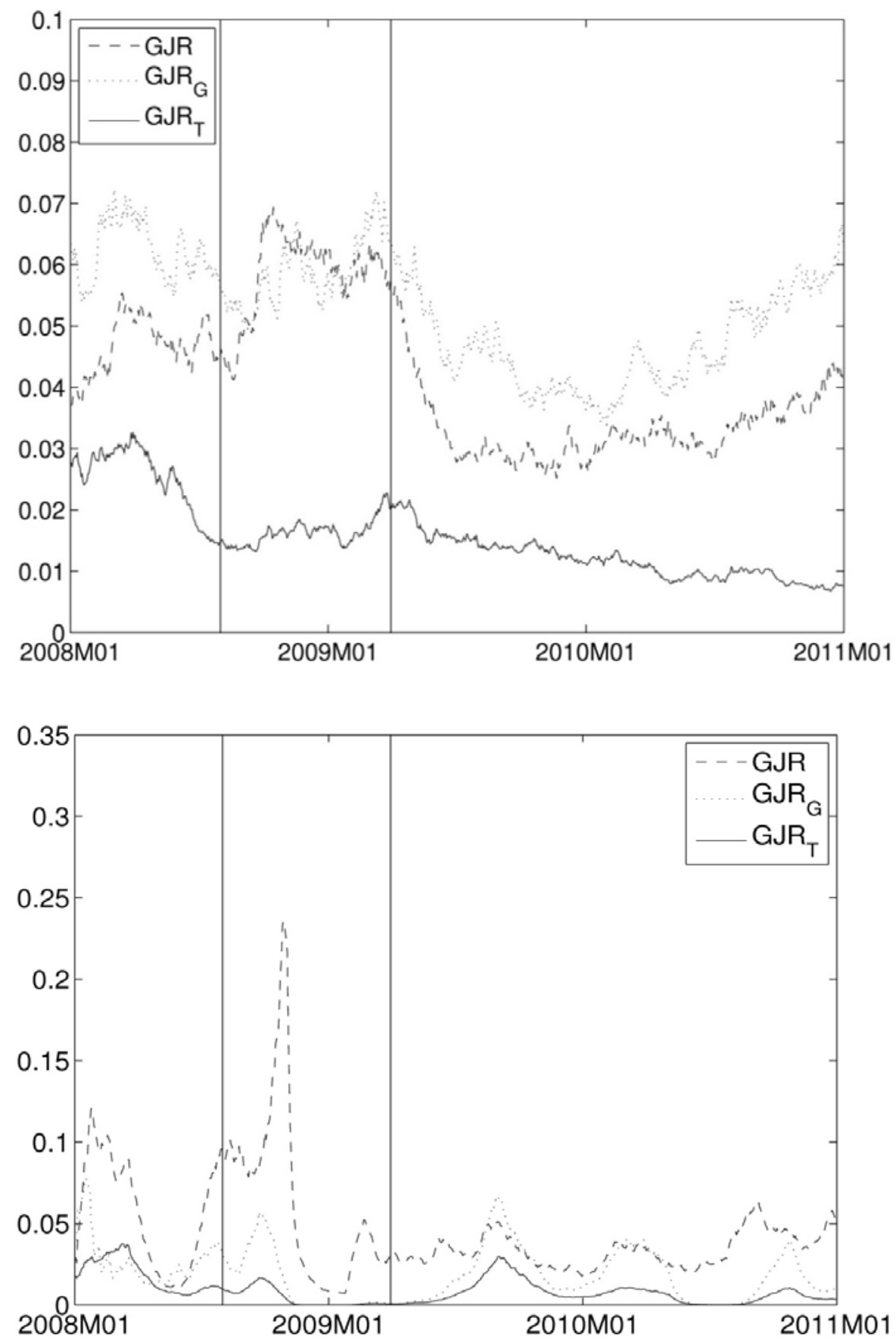

Figure 11

RW (upper) and CD (lower) strategies using filtered combination weights for three different GJR models. The vertical lines correspond to the possible starting (1 August 2008) and finishing (1 April 2009) dates of the GFC. 
Table 1

Basel Accord Penalty Zones

\begin{tabular}{|l|c|c|}
\hline Zone & Number of Violations & $k$ \\
\hline Green & 0 to 4 & 0.00 \\
\hline Yellow & 5 & 0.40 \\
& 6 & 0.50 \\
& 7 & 0.65 \\
& 9 & 0.75 \\
& $10+$ & 0.85 \\
\hline Red & \multicolumn{2}{|c|}{1.00} \\
\hline $\begin{array}{l}\text { Note: The number of violations is given for 250 business days. The penalty } \\
\text { structure under the Basel II Accord is specified for the number of violations } \\
\text { and not their magnitude, either individually or cumulatively. }\end{array}$ \\
\hline
\end{tabular}


Table 2

30-day Maturity VIX Futures Returns: Histogram and Descriptive Statistics 26 March 2004 - 10 January 2011

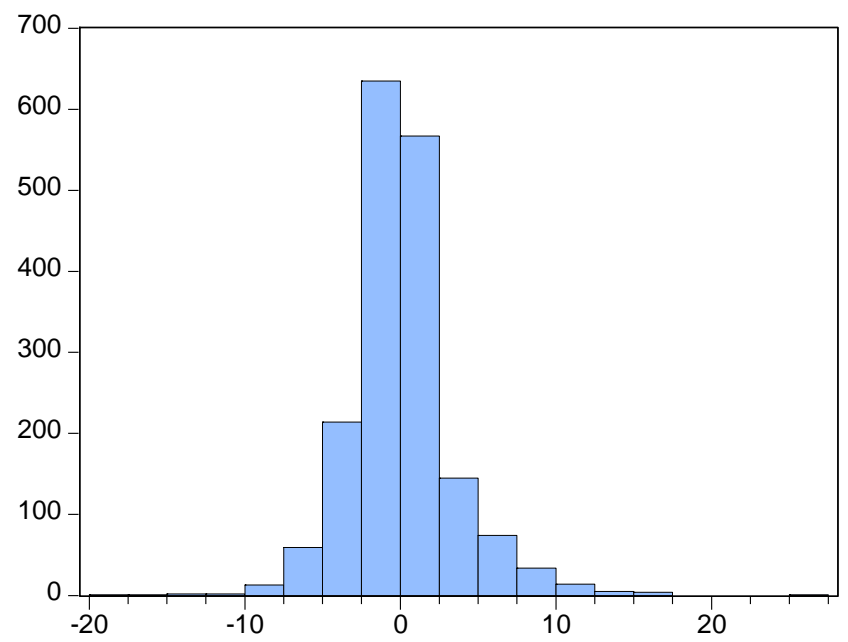

Series: VIX Futures

Sample 26/03/2004 10/01/2011

Observations 1771

Mean $\quad 0.001859$

Median $\quad-0.210453$

Maximum 25.81866

Minimum $\quad-19.92871$

Std. Dev. $\quad 3.412287$

Skewness $\quad 0.882711$

Kurtosis $\quad 8.210094$

Jarque-Bera 2233.068

Probability $\quad 0.000000$ 
Table 3

Tests for VIX Futures

\begin{tabular}{|c|c|c|}
\hline \multicolumn{3}{|c|}{ Tests for Equality of Means of VIX futures, t-test (p-value) } \\
\hline & During the crisis & After the crisis \\
\hline Before & $-16.73(0.00)$ & \\
\hline During & & $24.33(0.00)$ \\
\hline
\end{tabular}

Tests for Equality of Variances of VIX futures, F-test (P-value)

\begin{tabular}{|c|c|c|}
\hline & During the crisis & After the crisis \\
\hline Before & $21.93(0.00)$ & \\
\hline During & & $5.29(0.00)$ \\
\hline
\end{tabular}

Tests for Equality of distribution functions of VIX returns

Kolmogorov-Smirnov (P-value)

\begin{tabular}{|c|c|c|}
\hline & During the crisis & After the crisis \\
\hline Before & $0.153(0.053)$ & \\
\hline During & & $0.169(0.002)$ \\
\hline
\end{tabular}


Table 4

Descriptive Statistics for VIX Futures (Panel a) and VIX Futures Returns (Panel b)

Panel a

\begin{tabular}{|c|c|c|c|c|c|c|c|c|}
\hline AA_ID & Mean & Median & Max & Min. & Std. Dev. & Skew. & Kurt. & Obs. \\
\hline Before & 23.47 & 23.44 & 32.24 & 16.30 & 3.34 & 0.06 & 2.53 & 147 \\
\hline During & 45.57 & 45.69 & 80.86 & 18.810 & 15.66 & -0.06 & 2.31 & 151 \\
\hline After & 25.07 & 23.92 & 45.89 & 15.450 & 6.57 & 1.05 & 3.76 & 465 \\
\hline Whole period & 20.90 & 17.63 & 80.86 & 9.890 & 11.12 & 2.12 & 8.39 & 1711 \\
\hline
\end{tabular}

Panel b

\begin{tabular}{|c|c|c|c|c|c|c|c|c|}
\hline AA_ID & Mean & Median & Max & Min. & Std. Dev. & Skew. & Kurt. & Obs. \\
\hline Before & -0.010 & -0.264 & 16.252 & -8.555 & 3.344 & 0.934 & 6.061 & 153 \\
\hline During & 0.432 & 0.000 & 13.226 & -19.929 & 4.832 & -0.143 & 4.328 & 157 \\
\hline After & -0.158 & -0.449 & 13.402 & -13.469 & 3.601 & 0.839 & 4.882 & 480 \\
\hline Whole period & 0.002 & -0.210 & 25.818 & -19.929 & 3.412 & 0.883 & 8.210 & 1771 \\
\hline
\end{tabular}


Table 6

Sensitivity Analysis of Mean Capital Charges for the RW and CD

Strategies to the Choice of Window Size ( $\tau=10,50,100)$

\begin{tabular}{|c|c|c|c|}
\hline \multicolumn{3}{|c|}{ RW strategy } \\
\hline Period & $\tau=10$ & $\tau=50$ & $\tau=100$ \\
\hline $02 / 01 / 2008-01 / 08 / 2008$ & 24.358 & 23.747 & 23.677 \\
\hline $01 / 08 / 2008-01 / 04 / 2009$ & 33.522 & 33.126 & 32.459 \\
\hline $01 / 04 / 2009-16 / 03 / 2011$ & 28.009 & 27.843 & 27.426 \\
\hline \multicolumn{3}{|c|}{ CD strategy } \\
\hline $021 / 01 / 2008-01 / 08 / 2008$ & 23.811 & 23.747 & 23.387 \\
\hline $01 / 08 / 2008-01 / 04 / 2009$ & 30.724 & 33.125 & 30.606 \\
\hline $01 / 04 / 2009-16 / 03 / 2011$ & 27.252 & 27.844 & 27.094 \\
\hline
\end{tabular}

